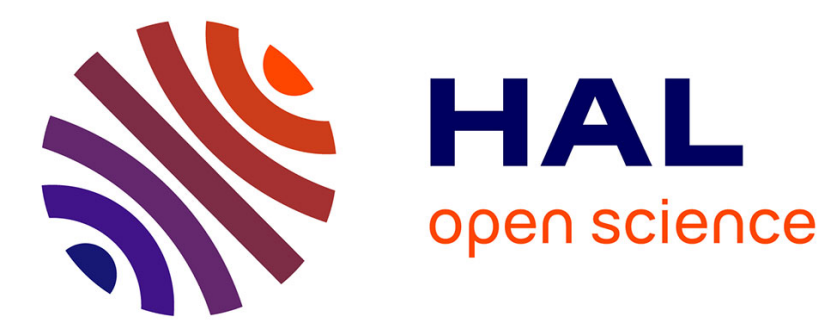

\title{
Lecture des tableaux parisiens : entre référence et imaginaire
}

\author{
Véronique Magri-Mourgues
}

\section{To cite this version:}

Véronique Magri-Mourgues. Lecture des tableaux parisiens: entre référence et imaginaire. L'information grammaticale, 2003, 96, pp.27-34. hal-01225991

\section{HAL Id: hal-01225991 \\ https://hal.science/hal-01225991}

Submitted on 10 Nov 2015

HAL is a multi-disciplinary open access archive for the deposit and dissemination of scientific research documents, whether they are published or not. The documents may come from teaching and research institutions in France or abroad, or from public or private research centers.
L'archive ouverte pluridisciplinaire HAL, est destinée au dépôt et à la diffusion de documents scientifiques de niveau recherche, publiés ou non, émanant des établissements d'enseignement et de recherche français ou étrangers, des laboratoires publics ou privés. 


\title{
LECTURE DES TABLEAUX PARISIENS : ENTRE RÉFÉRENCE ET IMAGINAIRE
}

\author{
Véronique Magri-Mourgues \\ Professeur des Universités \\ Univ. Nice Sophia Antipolis, membre de I'UCA \\ CNRS, BCL, UMR 7320 \\ http://www.unice.fr/bcl
}

La seconde section du recueil des Fleurs du Mal, Tableaux parisiens, n'apparaît que dans l'édition de 1861 et réunit huit poèmes de la section Spleen et Idéal de l'édition de 1857 ainsi que dix pièces parues dans les revues entre 1857 et 1861 . Une réunion en section de poèmes $a$ priori disparates suppose une cohérence à deux niveaux : comme nouvel élément dans l'architecture d'ensemble, la section doit justifier sa place au sein du recueil tout entier et doit construire son unité sur la cohérence entre les pièces qui la composent. Cette double cohérence peut se traduire en termes d'analogie: des ressemblances, thématiques ou formelles, sont censées rapprocher les dix-huit poèmes de Tableaux parisiens et, en même temps, ces points communs doivent être suffisamment spécifiques pour distinguer une section d'une autre. L'analogie se lit donc en termes d'attraction - répulsion ; l'outil d'analyse hypertextuelle que j'ai utilisé ${ }^{1}$ permet une première observation quantitative : cette section, comparée à l'ensemble du recueil, est celle qui présente le vocabulaire le plus varié et le plus grand nombre d'hapax ${ }^{2}$, ce qui peut grossièrement s'expliquer par la tonalité peut-être plus descriptive annoncée par le mot «tableaux» ou en tout cas par l'ancrage dans le référentiel, affirmé par l'adjectif relationnel. Cette particularité m'a conduite à choisir cette section comme objet d'une étude qui veut analyser la tension entre référentiel et construction imaginaire.

Le point de départ de l'analyse pourrait coïncider avec l'origine de toute vision, le « je », pivot autour duquel s'organise une expérience et se construit une vision du réel; analyser ses occurrences dans la section, c'est se situer d'emblée à un point de jonction entre intériorité et monde extérieur, entre référenciation et libre imagination. Le point de départ référentiel est comme un soubassement qui s'allège jusqu'à s'abstraire grâce à la mise en place du dispositif de l'imaginaire qui sera analysé au travers des procédés de l'abstraction temporelle et de l'abstraction nominale. L'imaginaire se surimpose même au référentiel pour s'y substituer par le processus de la métamorphose, qui s’organise autour de catégories ou de structures

\footnotetext{
${ }^{1}$ Le logiciel utilisé, Hyperbase, a été mis au point par Etienne Brunet, UMR 6039, Bases, Corpus, langage, 98 Bd Edouard Herriot, 06200 Nice.

${ }^{2}$ Mots qui n'apparaissent qu' une fois dans le recueil.
} 
syntaxiques particulières, comme la construction attributive ou le nom propre, et autour de structures discursives comme la comparaison et la métaphore.

Avant d'entrer dans le détail de ces deux processus, de l'imaginaire et de la métamorphose, l'élément paratextuel que sont les titres des pièces, à la fois à la marge du poème et en faisant partie, me paraît un nœud essentiel entre référence et imaginaire. Les titres fonctionnent comme une charnière entre la section tout entière et les poèmes, et comme un carrefour où se croisent les pièces mêmes ainsi que l'univers extratextuel du scripteur et le monde construit dans le livre. Ils ont donc une double vocation, qui se construit en amont d'une part et qui définit le poème comme fragment d'un ensemble plus vaste, qui se construit en aval d'autre part, comme prévision du poème à venir. Regard du poète sur son œuvre, les titres sont des balises posées sur le parcours d'une lecture, susceptibles de l'orienter ou de la désorienter. Seules deux pièces de la section en sont dépourvues, qui sont considérées comme les deux poèmes les plus intimistes, livrés ainsi dans toute leur pudique nudité, sans l'apparat d'aucune exhibition tapageuse. Le déterminant, point d'articulation entre le virtuel et l'actuel, révèle la portée référentielle des titres.

Deux pièces-invocations, $A$ une mendiante rousse, $A$ une passante, portent un titre qui prend la tournure d'une dédicace à un individu particulier par le biais de l'article indéfini. L'adjectif « rousse » restreint la portée du substantif pour le premier et accentue l'impression d'expérience vécue. Même si l'article « un » peut être doté d'une valeur généralisante, la particularité d'une expérience reste inscrite en filigrane.

D'autres titres utilisent l'article défini pluriel ou singulier ${ }^{3}$; « les » et «le » partagent « une condition existentielle d'unicité » soit d'un individu soit d'une classe ${ }^{4}$. L'interprétation des syntagmes ainsi définis oscille entre une acception particularisante pour laquelle l'unicité est justifiée par l'émergence du poème aussitôt après et une acception généralisante pour laquelle l'unicité est justifiée par le syntagme nominal lui-même. Trois poèmes successifs emploient l'article défini pluriel, Les Sept Vieillards, Les Petites Vieilles, Les Aveugles. Les deux premières pièces sont d'ailleurs senties comme formant un diptyque par Baudelaire qui les réunit sous l'appellation des « deux fantômes parisiens ». La présence de l'adjectif cardinal «sept », qui fonctionne comme déterminant complémentaire, contraint le choix de l'article défini tandis que l'adjectif qualificatif «petites » ne joue aucun rôle dans l'actualisation. L'interprétation générique est d'autant plus facile que le nom est dépourvu d'expansion : c'est le cas pour Les Aveugles, pièce que l'on a rapprochée d'un tableau de Breughel, auquel il emprunte aussi la portée symbolique. Avec «le», une étape est franchie : l'article défini, comme l'article indéfini, est bipolaire, oscillant entre une valeur particularisante et une valeur

\footnotetext{
${ }^{3}$ Les Sept Vieillards, Les Petites Vieilles, Les Aveugles

Le Soleil, Le Cygne, Le Squelette Laboureur, Le Crépuscule du soir, Le Jeu, L'Amour du mensonge, Le Crépuscule du Matin.

${ }^{4}$ Georges Kleiber, L’Article LE générique. La généricité sur le mode massif, Paris, Droz, 1989, p. 74.
} 
généralisante, mais « le » est plus enclin à faire entrer le syntagme dans l'ordre du symbolique : privilégié avec les noms qui peuvent recevoir une acception massive en langue, comme « amour » ou « jeu »- l'extension du nom est réduite dans le premier cas par un complément déterminatif - l'article «le » peut aussi bien déterminer des noms comptables. Selon Georges Kleiber, la combinaison «le » et un nom comptable peut aboutir dans certains contextes à la création d'un syntagme nominal massif, en ce sens qu'il peut présenter de manière homogène différentes occurrences, non distinguables. Le cas le plus probant de ce processus reste l'exemple de Le Cygne, érigé en représentant de sa classe et en véritable symbole ou «mythe étrange et fatal ».

Il resterait à parler de l'absence d'article dans quatre titres de la section : le premier poème Paysage appelle Danse macabre, Brumes et pluies et Rêve parisien : l'absence d'article répond aux normes de ce que certaines grammaires appellent les «étiquetages $»^{5}$. Cette absence manifeste un lien plus étroit entre le titre et le poème qu'il surplombe. Danse macabre est par exemple repris textuellement dans un vers du poème,

«Le branle universel de la danse macabre »: l'article défini maintient la valeur sémantique d'adjectif relationnel à «macabre »; l'adjectif « universel» accentue la portée générique du syntagme, l'ensemble renvoyant à un genre défini de tableau ou de sculpture allégorique du moyen âge.

L'outil statistique m'a permis de comparer l'emploi des déterminants dans le recueil tout entier : la section Tableaux parisiens est la seule qui se caractérise par l'emploi majoritaire du mot « les ». Le poids négligeable des pronoms personnels parmi les occurrences de « les », constaté après tri manuel, autorise à affirmer que l'article défini pluriel est spécifique de cette section du recueil. En tant que vecteur de la généricité, «les » présente un ensemble d'individus sous l'aspect hétérogène, autrement dit, il préserve l'idée de la pluralité sous l'unicité; cette observation est tout à fait en accord avec le titre même au pluriel de la section et avec son idée directrice : le kaleidoscope des images urbaines se résout en une vision unique de la ville à la fois moderne et intemporelle. Cette section est tout entière placée sous le signe de la tension entre l'un et le multiple, réalisée à différents niveaux et dont la présence majoritaire de l'article « les » est sans doute une manifestation. Un exemple probant est le poème Le Cygne et la longue chaîne des exilés qu'il égrène au fil des quatrains finals et des relatives nominales périphrastiques indéfinies :

A quiconque a perdu ce qui ne se retrouve

Jamais, jamais ! à ceux qui s'abreuvent de pleurs

Et tètent la Douleur comme une bonne louve !

\footnotetext{
${ }^{5}$ Voir par exemple Marc Wilmet, Grammaire critique du français, Paris, Bruxelles, Duculot, 1997, p. 152.
} 
La cohésion des maillons de cette chaîne syntaxique et référentielle est maintenue par la reprise anaphorique de la même locution verbale «je pense ». On peut évoquer aussi évidemment la répétition hallucinatoire des Sept Vieillards, qui repose sur l'expansion d'un singulier « un vieillard »; les six dernières strophes du poème jouent sur une alternance entre syntagmes nominaux singulier et pluriel : aux singuliers «son pareil», «ce jumeau centenaire», «ce sinistre vieillard », «sosie inexorable, ironique et fatal / Dégoûtant Phénix, fils et père de luimême », toujours en tête de vers, répondent les syntagmes pluriels « ces spectres baroques », «ces sept monstres hideux», tous deux réconciliés dans ce syntagme singulier et de sens collectif «au cortège infernal », cette fois occupant tout un second hémistiche, dans un vers conclusif.

Le jeu entre l'un et le multiple qui anime les visions mêmes du poète se réalise, au niveau énonciatif, entre le «je »du poète, unique, et les images plurielles du monde qu'il perçoit. Le troisième maillon du réseau énonciatif, le lecteur à la figure changeante, est un autre avatar de la multiplicité.

\section{Du « je » au monde}

De la vaporisation et de la centralisation du Moi. Tout est là. (Mon cour mis à nu, journal intime )

Alors que dans Les Fleurs du mal le pronom « je » est en déficit par rapport aux autres œuvres de Baudelaire 6 - les marques de première personne dans leur ensemble, pronoms personnels et adjectifs possessifs, sont en effet caractéristiques des Petits Poèmes en Prose - il est en revanche spécifique des Tableaux parisiens, comparés aux autres sections du recueil.

«Je » est le terme inaugural du premier poème de la section, sujet du verbe «veux », que certains linguistes considèrent comme semi-auxiliaire de volonté.

Je veux, pour composer chastement mes églogues,

Coucher auprès du ciel, comme les astrologues,

Et, voisin des clochers, écouter en rêvant

Leurs hymnes solennels emportés par le vent.

Toutefois, le groupe sujet-verbe est isolé dans la première mesure par la pause syntaxique qui permet d'insérer un complément circonstanciel de but avant l'intervention de l'infinitif complément au vers deux. Cette destructuration de ce qu'on aurait pu considérer comme une périphrase verbale laisse intacte toute la portée sémantique du verbe «vouloir » pour en faire la proclamation ferme d'une décision prise par le « je » qui se présente comme poète. Le poème suivant, Le Soleil, ne présente qu'une occurrence du pronom personnel «je », en tête du

\footnotetext{
${ }^{6}$ La base lemmatisée constituée par Etienne Brunet comprend Les Fleurs du Mal, les Petits Poèmes en prose, les
} Paradis artificiels, Le Fanfarlo, Le jeune Enchanteur. 
troisième vers, renforcée par l'adjectif «seul» à la césure développant la même idée de l'isolement du poète. Le soleil peut d'ailleurs être interprété comme métaphore du poète avec cette particularité de l'inversion de la comparaison attendue : c'est le soleil qui est comparé explicitement à un poète puis à un roi dans la suite du poème.

Quand, ainsi qu'un poète, il descend dans les villes [...](Le Soleil)

Les verbes régis par «je » sont révélateurs du rôle que le poète s'attribue dans les Tableaux parisiens : cette section se caractérise par l'emploi du paradigme du verbe «voir ${ }^{7}$. Sans qu'on puisse parler d'une véritable orientation descriptive de cette section - comme y invite cependant le titre - on pourrait dire que le poète se pose en témoin de la ville, de son décor et de sa faune, et par là de lui-même, soit qu'il s'identifie aux figures entrevues, soit qu'il en fasse une lecture allégorique, soit qu'il s'observe lui-même dans un dédoublement fantasmatique.

Le verbe «voir » garde son sens de verbe de perception visuelle quand il régit lui-même une proposition infinitive dans l'exemple suivant :

Lorsque la bûche siffle et chante, si le soir

Calme, dans le fauteuil je la voyais s'asseoir (La servante au grand cour)

Ou dans celui-ci, si on accepte l'idée que Baudelaire s'inspire d'un tableau allégorique authentique pour ce poème :

Quand je te vois passer, ô ma chère indolente (L'Amour du mensonge)

La polysémie du verbe « voir » autorise ce dernier à dénoter aussi bien la vision au sens concret et physique du terme que la vision de l'esprit qui fait basculer dans l'imaginaire et le rêve. Toutes les occurrences du verbe « voir » sont ambivalentes dans notre corpus. Il en est de même du mot «tableau» pour ses trois occurrences dans les poèmes de la section. Ce mot est complément essentiel du verbe «voir» ou rapproché du verbe «entrevoir» pour les deux premiers exemples :

Et lorsque j'entrevois un fantôme débile

Traversant de Paris le fourmillant tableau (Les Petites Vieilles)

Voilà le noir tableau qu'en un rêve nocturne

\footnotetext{
${ }^{7}$ Deux formes sont spécifiques « vois » et « vis » par rapport à l'ensemble du recueil. La forme « vis » est toutefois amphibologique appartenant au paradigme des verbes « voir» et « vivre». Une occurrence des Tableaux parisiens, correspond au verbe « vivre».
} 
Je vis se dérouler sous mon œil clairvoyant. (Le Jeu)

Et, peintre fier de mon génie,

Je savourais dans mon tableau

L'enivrante monotonie

Du métal, du marbre et de l'eau. (Rêve parisien)

Si, pour la première occurrence, le poème présente une vision fantasmatique du réel, il fait basculer clairement dans l'onirique, au sens premier du terme, pour les deux suivantes. Dans Rêve parisien, la répétition de la forme présentative minimale «c'était » au singulier ou au pluriel en tête de vers pour les deux premières occurrences, en position de contre-rejet externe pour la troisième, introduit dans l'univers enchanté et surréel du rêve ${ }^{8}$.

Les premières occurrences du verbe « voir », dans Paysage, sont conjuguées au futur qui ajoute ses valeurs modales au signifié du verbe : la vision est ici rêvée - au sens d'imaginée. La proximité de la forme « rêverai » qui glisse résolument du côté de l'onirique et qui est en outre mise en valeur à une place-clé du vers, la césure, ne fait que renforcer le caractère imaginaire de la vision. On retrouve une forme de verbe «voir» au futur mais à la forme interrogative dans $A$ une passante, et dans un contexte particulier :

Ne te verrai-je plus que dans l'éternité ?

Dans Le Cygne, les syntagmes « je vis » ou « je vois » introduisent un souvenir qui se superpose au spectacle réel et cette fois, en relation avec le syntagme « je pense » construit en anaphore qui rythme les dernières strophes. Des variantes du verbe «voir», comme « je guette » (Les Petites Vieilles), affirment la posture du poète observateur. Le verbe « entrevoir », quant à lui, compte une occurrence dans Les Petites Vieilles et ajoute le sème d'incertitude.

Lors de ses hallucinations, le poète se dédouble et devient observateur de lui-même : ce regard réflexif est syntaxiquement exprimé par les tournures pronominales avec attribut :

Moi-même, dans un coin de l'antre taciturne,

Je me vis accoudé, froid, muet, enviant,

[...] Le Jeu

8 «C'était un palais infini $[\ldots]$

C'étaient des pierres inouïes

Et des flots magiques ; c'étaient

D'immenses glaces éblouies

Par tout ce qu'elle reflétaient !» 
Le poète est un témoin mais qui devient acteur de la ville aussi et qui peut être observé luimême par la cité personnifiée dans ce poème essentiel dans notre perspective, Les Aveugles :

\author{
Ils traversent ainsi le noir illimité, \\ Ce frère du silence éternel, $O$ cité ! \\ Pendant qu'autour de nous tu chantes, ris et beugles,
}

Eprise du plaisir jusqu'à l'atrocité,

Vois ! je me traîne aussi ! mais, plus qu'eux hébété. (Les Aveugles)

Cette oscillation $\mathrm{du}$ « je » au monde qui traduit constamment une vision du réel se replie en vision du monde intérieur, en véritable huis-clos dans plusieurs poèmes. Deux pièces sont structurées sur une dynamique du resserrement : Paysage d'abord ; cette pièce liminaire signale d'emblée le repli du poète sur lui-même. Le vers au futur / « Je verrai les printemps, les étés, les automnes » / appelle la rime en «monotones » qui ne fait que confirmer la continuité et la régularité signifiée par l'énumération des trois saisons au pluriel tandis que l' « hiver » se trouve inséré dans un complément circonstanciel de temps en position thématique. Le vers suivant véhicule le prédicat et signale le repli du poète dans un autre univers, intérieur celui-là. Ces indications temporelles sont comme annulées par la reprise, en fin de poème, du mot «Printemps » avec majuscule et au singulier cette fois, pour signifier le passage à l'ordre du symbolique, et la bascule complète dans l'ordre du rêve. Le contre-rejet («cette volupté / d'évoquer »), la remotivation du sens étymologique du verbe «évoquer » et les deux vers suivants et derniers du poème insistent sur la surimposition du monde imaginaire. Le choc de l'article indéfini ( « un soleil, une tiède atmosphère ») et des adjectifs possessifs qui permettent la désignation métonymique du poète (« mon cœur », «mes pensers brûlants ») de même que le rythme ternaire institué par la reprise de la préposition «de» qui introduit un infinitif dénotant un acte créateur (« évoquer, tirer, faire ») martèlent ce pouvoir démiurgique du poète. Le Crépuscule du Soir est le seul poème de la section avec $A$ une passante où des notations auditives sont faites :

On entend çà et là les cuisines siffler,

Les théâtres glapir, les orchestres ronfler ;

alors que la section est consacrée - comme son titre paraît l'annoncer - à un spectacle visuel de Paris. Cependant, le même mouvement structure le poème; le second volet signale l'enfermement du poète en lui-même comme le note l'apostrophe qui personnifie son âme :

Recueille-toi, mon âme, en ce grave moment, 
Et ferme ton oreille à ce rugissement.

D'autres poèmes sont dépourvus d'occurrences de «je» et signalent un mouvement symétrique, celui d'une extension au-delà $d u$ «je » du poète ; le premier poème qui en est exempt est Le Squelette laboureur : le « je » est d'abord relayé par le pronom personnel indéfini « on », sujet du verbe « voit », puis par les formes du pluriel «nous » qui prend sa plus grande extension, dénotant l'Humanité tout entière. La même absence caractérise Le Crépuscule du Soir palliée cette fois par une adresse à l'âme du poète, personnifiée. L'emploi de pronoms personnels spécifiques, de l'impersonnel, de relatives substantives ou de l'infinitif contribue à ce mouvement d'extension grâce à la dilatation de la personne du « je » ou à son effacement. L'infinitif, par exemple, estompe l'ancrage référentiel précis, personnel ou temporel. Son emploi est illustré par le poème Paysage : un infinitif circonstanciel s'interpose dès l'émergence $\mathrm{du}$ « je » et retarde l'apparition de l'infinitif «coucher » en position forte, en début du vers deux, construit en parallèle avec le second infinitif de même fonction en début d'hémistiche au vers trois. Une majorité d'infinitifs occupe le poème : la locution impersonnelle « il est doux » entrâne un groupe infinitif, sujet thématique, qui lui-même appelle une cascade d'infinitifs, pivots de propositions infinitives, toujours placés à des places-clé du vers («naître, monter, verser »). L'infinitif est encore relayé par l'autre mode impersonnel du participe présent dont l'aspect sécant est en concordance avec le sens lexical des verbes, («rêvant », «pleurant», «chantant »). Deux vers de ce poème, qui suivent le même patron rythmique et syntaxique, paraissent constituer avec les deux vers qui les encadrent un quatrain quasi autonome, comme une séquence à tendance descriptive, appuyée sur les participes présents qui suspendent le temps.

\footnotetext{
Alors je rêverai des horizons bleuâtres,

Des jardins, des jets d'eau pleurant dans les albâtres,

Des baisers, des oiseaux chantant soir et matin,

Et tout ce que l'Idylle a de plus enfantin.
}

L'infinitif permet l'extension d'une expérience parce qu'il joue sur un pivot du dispositif de l'imaginaire qui est l'abstraction temporelle.

\section{Le dispositif de l'imaginaire}

«Tout l'univers visible n'est qu'un magasin d'images et de signes auxquels l'imagination donnera une place et une valeur relatives ; c'est une espèce de pâture que l'imagination doit digérer et transformer » (Curiosités esthétiques, Salon de 1859, Le Gouvernement de l'imagination) 
La catégorie du temps est sensible au passage du référentiel à l'imaginaire. Plusieurs pièces se situent d'emblée dans le présent générique qui suspend l'inscription du procès dans une temporalité repérable : «le présent génère sa propre actualité, transporte avec lui son repère, et gagne une autonomie par rapport à son environnement ${ }^{9}$. Les énoncés gagnent ainsi en abstraction, en ce sens qu'ils apparaissent détachés des circonstances spatio-temporelles ${ }^{10}$. Dans le poème Le Soleil, le présent se colore d'une valeur itérative ; d'autres poèmes se placent résolument dans l'intemporel et le générique comme Les Aveugles ou Le Crépuscule du Soir. Plus révélateurs de ce mouvement d'abstraction temporelle sont les poèmes où des circonstants temporels sont d'abord clairement notés comme cadre d'une expérience authentique du poète. Ce cadre vole ensuite en éclats sous la pression de l'abstraction et de l'imaginaire.

Le Cygne est une pièce construite sur l'opposition entre deux époques, dénotée par l'alternance entre le présent de l'indicatif et le passé simple. Le passé simple, tiroir verbal du fait passé et avéré, se trouve inséré dans un environnement imagé, dans une sorte d'expansion de l'imaginaire développé à partir de l'adverbe de lieu « là »-palimpseste, où se superposent deux époques, et qui sert de tremplin à l'émergence du souvenir. Le poème se termine éloquemment sur l'allégorie du Travail.

Le Crépuscule du Soir s'ouvre sur le présentatif déictique, « voici », censé ancrer l'énonciation dans la situation du locuteur ; en fait, le présent de « il vient » du vers deux qui peut passer pour présent actuel se mue très vite en présent intemporel dès que l'envol de l'imaginaire est pris, appuyé sur le syntagme défini générique, «l'homme impatient». L'écho ironique entre «en bête fauve » et « à pas de loup » oblige à une relecture de l'expression lexicalisée et par là même à une réinterprétation généralisante du présentatif « voici ».

Le Crépuscule du Matin joue sur une autre alternance, entre l'imparfait de l'indicatif et le présent. On sait que l'imparfait a une vocation aspectuelle plus que temporelle, ce qui le rend apte à convenir à n'importe quel repérage temporel. Le seul tiroir verbal qui lui réponde dans ce poème est le présent gnomique qui brouille toute référence temporelle précise. Si l'imparfait paraît situer les procès dans un continuum temporel passé, en introduisant le lecteur in medias res, il n'empêche pas « une mer de brouillards » de se répandre sur la temporalité du poème comme sur son décor. Le présent joue en faveur d'une dilution temporelle. Le syntagme présentatif «c'était l'heure » qui compte deux occurrences réparties dans deux strophes successives et qui rythme la première strophe par la reprise de l'adverbe relatif « où » (vers 3 , $5,7)$ s'apparente aux formules mystérieuses qui ouvrent un monde imaginaire ou mythique, dont les lois particulières sont acceptées d'emblée par celui qui y pénètre.

\footnotetext{
9 A. Jaubert, 2001, p. 67.

10 Un syntagme générique représente un emploi du nom qui peut être considéré comme abstrait, non pas parce que le type d'occurrences correspondant au nom est abstrait, mais parce que le référent dénoté dans cet emploi n'est pas un référent factuel, contingent. (Voir G. Kleiber, 1994, p. 51-52).
} 
Le glissement du présent actuel au présent générique ${ }^{11}$ illustre encore cette explosion du cadre temporel. Les exemples les plus probants sont les poèmes qui développent la structure de l'apostrophe ${ }^{12}$, fréquente dans Les Fleurs $d u$ Mal ; des apostrophes ponctuelles apparaissent parfois comme dans Le Cygne, Les Petites Vieilles où le « je » interroge le lecteur potentiel ou s'adresse aux figures entrevues. Certains poèmes en revanche sont construits sur une situation dialogique étendue. Dans ces poèmes-apostrophe ou invocation, le présent est censé être défini par le contexte d'énonciation même. La structure dialogique installe d'emblée le poème dans un pseudo-réalisme.

Dans A une Mendiante rousse, le lecteur est cependant alerté par la majuscule du mot « mendiante ${ }^{13}$ dès le titre, qui confere une portée emblématique à la figure ; cette mendiante devient comme une incarnation de la beauté - ce que souligne la reprise du terme «beauté » à la fin de la première strophe et en fin de poème : d'une acception neutre, le poète est passé à une acception métonymique contenue dans l'apostrophe. De fait, le présent employé glisse dans la sphère du générique

A une passante est un autre poème apostrophe. Là encore, l'article indéfini oscille entre une portée particularisante et une portée généralisante. Mais le seul pronom «tu», marqueur déictique, a un rôle locutoire, désignant l'allocutaire comme référent, mais reste muet sur la référence sémantique du pronom. La structure dialogique, de fait, s'abstrait et s'élève au schéma intemporel de l'échange entre un « je » et un « tu » malléables à souhait ${ }^{14}$.

Danse macabre est plus original quant à l'emploi qui est fait de la structure dialogique : Le passage de la troisième personne à la seconde structure le poème et invite à un partage des quatrains : Les quatre premiers jouent sur l'ambiguité du délocuté non nommé en adoptant une tonalité descriptive ; le cinquième quatrain l'identifie par le biais de l'apostrophe à la césure, «grand squelette», tandis que les quatrains suivants adoptent une démarche réflexive : les modalités de phrase sont plus volontiers exclamatives et interrogatives; le rythme est plus heurté : par exemple dans les vers suivants,

Viens-tu troubler, avec ta puissante grimace,

La fête de la Vie? ou quelque vieux désir,

11 Voir P. Guiraud, Essais de stylistique, Paris, Klincksieck, 1985 qui propose une comparaison du poème en prose, La Chevelure, et de Parfum exotique fondée sur l'emploi du présent, générique dans le premier cas, actuel dans le second.

12 Voir M.-H. Prat, « L'apostrophe dans Les Fleurs du Mal », in L’Information grammaticale, n 40, janvier 1989.

13 A supposer qu'elle soit le fait de Baudelaire même.

14 Voir Georges Kleiber, Dialogue, deixis et anaphore, p. 161-181, in Le Dialogique, colloque international sur les formes philosophiques, linguistiques, littéraires, et cognitives du dialogue, Berlin, Peter Lang, 1997. 
L'enjambement est entravé par le complément circonstanciel à la fin du premier vers tandis que le second vers se caractérise par une pause syntaxique marquée à la césure. Le poème se termine sur une prosopopée fantastique qui donne la parole au squelette danseur ${ }^{15}$.

L'Amour du mensonge présente une situation similaire: Le référent $\mathrm{du}$ «tu » est rendu problématique ; le référent sémantique reste énigmatique et l'apostrophe métonymique «ô ma chère indolente »n'y change rien, même si des échos lexicaux rapprochent ce poème du précédent ; «regard profond » fait écho à la métaphore nominale « le gouffre de tes yeux », remotivant le sens propre de l'adjectif «profond»dans l'expression lexicalisée «regard profond $\gg$.

Dans Brumes et pluies, la structure dialogique est, cette fois, d'emblée placée sous le signe de la fiction personnifiante puisque le premier quatrain de ce sonnet s'ouvre sur une adresse à un non animé, les saisons. L'apostrophe est reprise dans le premier tercet avec une légère variante, assurant la cohésion entre les strophes. Le dialogue se dévoile clairement comme artifice rhétorique.

Le mouvement vers l'abstraction temporelle se réalise aussi via le substantif, catégorie qui est dégagée en langue de toute contrainte situationnelle: Le décompte statistique me permet d'affirmer que le substantif est spécifique du recueil des Fleurs du Mal comparé aux autres œuvres de Baudelaire du corpus, avec un avantage particulier pour le substantif masculin singulier. La métonymie de l'abstraction est d'autre part un procédé récurrent en poésie qu'on retrouve dans Les Fleurs du Mal; en voici un exemple :

Il est doux, à travers les brumes, de voir naître [...]

Et la lune verser son pâle enchantement. (Paysage)

Le raccourci nominal abstrait «enchantement», qui réduit un syntagme nominal avec expansion adjectivale comme «sa pâle lumière enchantée », double la synesthésie qui assimile la lumière à un élément liquide - par le biais du verbe «verser» dont un prédicat type est un liquide - d'un entrelacs entre concret et abstrait. Ce mouvement d'abstraction fonctionne comme un outil d'appropriation du réel : l'action se cristallise dans un substantif abstrait.

L'oscillation permanente entre référentiel et imaginaire structure en profondeur les poèmes.

Les deux strophes de longueur inégale qui composent le poème Paysage s'ouvrent, la première sur un « je » particulier, l'autre sur une formule impersonnelle « il est doux » qui fait quitter le socle du présent actuel pour la sphère de l'intemporel, du générique ; les deux volets marquent l'extension du singulier au générique. Néanmoins, des récurrences lexicales maintiennent la

15 Voir Henk Nuiten et Maurice Geelen, Baudelaire et le cliché, Zeitschrift für französische Sprache und Literatur, Beiheft 17 : cliché nominal du «trouble-fête » remotivé par le changement de catégorie grammaticale et par disjonction du verbe et de son complément d'objet. 
cohésion entre eux deux : le syntagme «je verrai » ainsi que le verbe «rêver » à l'infinitif d'abord, au futur et en écho aux formes du même tiroir verbal dans la seconde strophe ensuite, « je rêverai ». De la construction transitive indirecte «rêver d'éternité » à la construction transitive directe « je rêverai » qui régit une suite de compléments d'objet directs comme le révèle le dernier terme de l'énumération, «je rêverai [...] tout ce que l'Idylle a de plus enfantin », une nuance de sens est manifeste : équivalent de « souhaiter, désirer » d'abord, le verbe devient synonyme d' « imaginer », de «créer » et assure le glissement dans la sphère de l'imaginaire. La valeur modale des futurs est de même encouragée par le cotexte.

Le poème pastiche, A une mendiante rousse, attire l'attention par le choix d'un mètre court et impair pour les trois premiers vers de chaque strophe qui contraste avec les alexandrins des deux poèmes précédents. Le jeu entre l'impair (le mètre) et le pair (les quatrains) est redoublé par le jeu entre l'imaginaire et le référentiel. Le poème s'articule autour du connecteur « cependant » qui signale la retombée dans la triste réalité après l'envol imaginaire qui décrit la métamorphose tout hypothétique de la mendiante rêvée par le biais du subjonctif de souhait et du conditionnel ; chaque quatrain est construit sur un contraste similaire. Le tétramètre final des deux premières strophes crée un effet de chute grâce au procédé rythmique de discordance entre mètre et syntaxe. La rupture est consacrée avec le connecteur « cependant » et le tiret, qui prennent une valeur concessive.

Dans Rêve parisien encore, au mètre court aussi mais pair (octosyllabe), l'opposition du rêve et de la réalité est clairement manifestée par la distribution du poème en deux volets : à l'expansion onirique et éclatante de lumière succèdent de brèves notations dysphoriques.

Le poème Le Cygne bascule, quant à lui, du côté du souvenir et de l'imaginaire ; le seul ancrage référentiel est la circonstancielle « Comme je traversais le nouveau Carrousel » qui s'ouvre sur un introducteur moins univoque que la conjonction «quand». L'emploi de l'imparfait de l'indicatif, par l'aspect et les connotations modales hypothétiques que ce tiroir verbal peut véhiculer, convient à l'envolée dans le monde imaginaire. Le poème est en effet construit ensuite sur une succession d'images développées ou à peine esquissées, le cygne, Andromaque, la négresse, les matelots, les captifs, les vaincus.

Suit un groupe de trois poèmes qui sont liés par des caractéristiques communes, la disposition croisée des rimes et une présentation énigmatique similaire.

Le Jeu adopte une structure qu'on pourrait qualifier d'à rebours : Le poème commence sur une suite de vers de tonalité descriptive sans aucun ancrage temporel explicite puisqu' aucune forme verbale conjuguée n'apparaît comme centre de proposition principale. Seule une forme de présent sert de pivot à une relative, sans qu'on puisse en déterminer la portée. Une énumération de syntagmes nominaux s'organise autour d'indications spatiales variées qui rythment les deux premières séquences en suite de quatrains sans que la phrase soit interrompue par une pause forte : «Dans des fauteuils fanés », «Autour des verts tapis », «sous de sales plafonds », 
compléments qui n'introduisent que des formes en -ant elles aussi sans capacité d'actualisation, «minaudant, faisant, fouillant, projetant». C'est seulement le présentatif «voilà », anaphorique, qui désigne les vers précédents comme « un rêve nocturne » et oblige à une relecture du poème.

Le poème suivant Danse macabre, dont les rimes sont également croisées, joue sur un même effet de surprise puisque seul le pronom énigmatique «elle»sans référent explicité apparaît d'abord comme thème du poème. Des indices font ensuite écho au titre du poème: «les funèbres appas », ses yeux « faits de vide et de ténèbres » avant l'apostrophe révélatrice « grand squelette ».

Le poème L'Amour du mensonge adopte une structure dialogique mais reste tout autant énigmatique que les précédents. L'apostrophe métonymique «ô ma chère indolente » n'identifie pas l'interlocutrice et la série d'attributs sous une forme interrogative contribue à brouiller les repères en convoquant plusieurs syntagmes qui peuvent être interprétés comme métaphoriques.

\footnotetext{
«Es-tu le fruit d'automne aux saveurs souveraines?

Es-tu vase funèbre attendant quelques pleurs,

Parfum qui fait rêver aux oasis lointaines,

Oreiller caressant, ou corbeille de fleurs ?»
}

Les Sept vieillards commence par une tournure anecdotique : un circonstant de temps « un matin» singulatif se déroule lentement au gré d'autres indications circonstancielles (« cependant que... et que ») qui débordent même d'une strophe à l'autre : le rythme est ralenti par l'insertion de segments explicatifs, la relative adjective d'abord, l'apposition phrastique ensuite ainsi que par les deux participes présents qui font attendre le complément d'objet. Ce ralentissement favorise l'irruption du vieillard à la quatrième strophe, dramatisée par la pause syntaxique marquée après le groupe verbal « m'apparut », tandis que la césure est estompée par l'enjambement interne entre le verbe et son complément d'objet. Le rejet du circonstanciel «dans le fiel» reproduit le même rythme linguistique dans le vers suivant comme pour accentuer le choc de l'apparition et aligne cette strophe sur la strophe précédente où le premier vers se distingue aussi par l'isolement d'une mesure de trois syllabes. Après une première strophe d'avertissement, la syntaxe regroupe les quatrains deux par deux. Cependant à partir de l'apparition du « pareil »du vieillard, chaque strophe se clôt sur une pause forte, contenant dans des cadres étroits la crise hallucinatoire à son paroxysme.

La bascule du référentiel à l'imaginaire structure plusieurs poèmes de la section et repose essentiellement sur une dynamique d'abstraction temporelle : la pression de l'onirique ou de l'hallucinatoire fait exploser les cadres d'énonciation supposés les mieux ancrés, comme ceux 
des poèmes dialogiques. Ce glissement du référentiel à l'imaginaire se trouve reproduit au niveau microstructural des poèmes, au niveau de la phrase et du vers. La métamorphose du référent peut s'opérer à la faveur de tours syntaxiques particuliers, par exemple attributifs, ou de figures discursives spécifiques.

\section{Le processus de la métamorphose}

Dans un emploi particulier, le verbe «faire » sert la dynamique de l'imaginaire et devient un instrument de la métamorphose du réel. Le verbe «faire », factitif, suivi d'un infinitif, forme avec ce dernier une unité lexicale et grammaticale qui peut être appréhendée comme périphrase actancielle fonctionnant par ajout d'un actant. La cohésion syntaxique et sémantique accomplie - confirmée notamment par l'invariabilité du participe « fait » dans le cas d'une forme verbale composée - paraît favoriser l'émergence d'un noyau verbal autonome apte à modifier une situation. Pour deux occurrences, l'infinitif est le verbe «rêver » sans agent exprimé soulignant le point de départ référentiel, tremplin pour l'imaginaire :

Et les grands ciels qui font rêver d'éternité. (Paysage)

Parfum qui fait rêver aux oasis lointaines. (L'Amour du mensonge)

Le parallélisme syntaxique - remarquable en dépit de la variation de la préposition régie par le verbe qui ne paraît pas induire de différence sémantique notable - dans ces deux occurrences favorise le rapprochement entre les compléments du verbe, une distribution complémentaire ou une équivalence entre les catégories temporelle et spatiale. Une différence de rythme est cependant remarquable : pour la première occurrence, un contre-rejet interne isole le semiauxiliaire «fait » et redouble, par la structure prosodique, le signifié du vers. Dans le second exemple, si on peut encore parler d'un enjambement interne, la pause ménagée entre les hémistiches préserve la cohérence syntaxique de la périphrase verbale et joue sur la fluidité du vers.

Deux autres occurrences de périphrase factitive explicitent l'agent de l'infinitif qui subit aussi le pouvoir bénéfique du sujet grammatical : le soleil et le regard de la passante.

Il [le soleil] fait s'évaporer les soucis vers le ciel (Le Soleil)

Fugitive beauté

Dont le regard m'a fait soudainement renaître. (A une passante) 
Lorsque c'est le « je » qui est sujet grammatical de la périphrase, celle-ci insiste sur le pouvoir démiurgique du poète même, notamment dans le poème Rêve parisien tout entier placé sous l'influence d'un rêve nocturne et cependant dirigé par le poète.

Architecte de mes féeries,

Je faisais, à ma volonté,

Sous un tunnel de pierreries

Passer un océan dompté. (Rêve parisien)

La distribution des membres de la périphrase sur deux vers non successifs leur rend une part d'autonomie et l'ampleur du rythme ainsi créée exprime comme une jubilation créatrice libérée de toute entrave.

Un autre emploi du verbe «faire », que l'on peut analyser comme attributif, opère une métamorphose d'un élément premier, complément d'objet, à un élément métaphorique, attribut de ce complément. Deux occurrences peuvent être relevées qui ont par ailleurs en commun d'être associées à des procédés prosodiques similaires :
Car je serai plongé dans cette volupté
D’évoquer le Printemps avec ma volonté,
De tirer un soleil de mon cœur, et de faire
De mes pensers brûlants une tiède atmosphère. (Paysage)

Le contre-rejet externe en écho au vers précédent place à la rime le verbe «faire». Dans Paysage, les figures syntaxiques de la métamorphose se multiplient ; la formule « tirer un soleil de mon cœur », elle aussi complément du syntagme cataphorique «cette volupté », décrit un même processus créateur, rehaussé par le chiasme sémantique, le résultat précédant le point de départ référentiel. Un contre-rejet interne cette fois sépare le verbe « faire » de ses compléments dans un autre exemple :

\footnotetext{
Toutes m'enivrent; mais parmi ces êtres frêles

Il en est qui, faisant de la douleur un miel,

Ont dit au Dévouement qui leur prêtait ses ailes:

Hippogriffe puissant, mène-moi jusqu'au ciel ! (Les Petites vieilles)
}

Le contre-rejet - qu'il soit interne ou externe - a pour effet de rapprocher dans un même hémistiche ou dans un même vers les syntagmes nominaux, le point de départ référentiel, le résultat métaphorique. 
Une variante peut être relevée qui utilise cette fois la préposition «avec » rendant plus problématique l'analyse en attribut :

Toutes auraient pu faire un fleuve avec leurs pleurs! (Les Petites vieilles)

La particularité de cette occurrence est d'antéposer le complément direct, c'est-à-dire l'élément métaphorique ou allotope.

La catégorie du nom et en particulier du nom propre est au centre des échanges entre référence et imaginaire. L'ancrage référentiel est par nature associé au nom propre censé dénoter un référent unique. Cette particularité est manifeste pour les dédicataires des poèmes, placés en exergue, donc en marge du poème, qui signalent l'intrusion sans ambiguïté du réel dans la fiction en tissant une relation entre le poète et des personnes.

Ces dédicaces ne manifestent pas de travail sur le nom propre. Insérés dans le corps du texte même, les noms propres de lieu esquissent une topographie parisienne (la Seine, Paris, Le nouveau Carrousel) tandis que les noms propres de personnes dessinent un autre parcours temporel et intertextuel qui irise les poèmes. Le travail de l'imaginaire se manifeste par le pluriel $^{16}$ qui affecte quelquefois les noms propres («des Ganges » in Rêve parisien, «Eves octogénaires » in Les Petites vieilles, « lovelaces ${ }^{17}$ chenus » in Danse macabre) tablant sur le procédé de l'antonomase, par métaphore. Les Petites vieilles fournit encore deux exemples de noms propres, dans la même position d'apostrophe, au singulier sans doute cette fois : Eponine ou Laïs. Inversement, des noms communs reçoivent une majuscule : c'est alors le processus de formation du symbole qui est lancé comme pour Le Squelette laboureur, ou celui de l'allégorie ${ }^{18}$ qui fait basculer pleinement dans le monde imaginaire. L'allégorie est parfois développée, comme celle de l'Infortune dans Les Petites vieilles :

Ces yeux mystérieux ont d'invincibles charmes

Pour celui que l'austère Infortune allaita.

On retrouve une image similaire dans Le Cygne, qui repose sur une autre allégorie, celle de la Douleur, et sur l'allusion à la louve qui allaita Romus et Rémulus ; l'entrelacement des images permet de tresser un réseau de relations entre les pièces de la section :

\footnotetext{
16 Voir M.-N. Gary-Prieur, Les noms propres et le nombre, Paris, CNRS éditions, 2001.

17 «J'appelle vos yeux sur Lovelaces. Si c'est un substantif, petit l, et un s final. Si c'est un nom propre que nous généralisons occasionnellement, grand 1 et pas d's. [...] En somme, Lovelace est presque un substantif de conversation. J'opine pour le petit 1 et le pluriel » (A Alphonse de Calonne, 1859, cité in Les Fleurs du Mal, (1861), introduction de Claude Pichois, NRF Gallimard, 1972).

18 Voir P. Labarthe, Baudelaire et l'histoire de l'allégorie, Paris, Droz, 1999.
} 
A quiconque a perdu ce qui ne se retrouve

Jamais, jamais ! à ceux qui s'abreuvent de pleurs

Et tètent la Douleur comme une bonne louve !

De manière plus traditionnelle, les outils de l'analogie majeurs comme la comparaison et la métaphore sont des instruments de choix pour la métamorphose du réel.

La rhétorique a associé comparaison et métaphore parce qu'elles sont censées illustrer toutes les deux une relation d'analogie et par là-même supposent toutes deux un énoncé fictionnel ; cependant, il n'est plus à démontrer qu'elles correspondent en fait à deux modes d'approche du réel différents. Pour les recherches les plus récentes sur le sujet, Catherine Détrie ${ }^{19}$ envisage d'opposer les deux processus en termes d'être pour et d'être comme. Elle resitue comparaison et métaphore dans leur rapport au réel ou plutôt les envisage comme outils pour rendre compte de ce que le locuteur perçoit du réel. Une approche syntaxique et cognitive des procédés pourrait être menée, de même qu'une étude des interrelations ${ }^{20}$ entre comparaison et métaphore dans un même poème révèle les liens étroits entre les deux procédés et leur rôle dans la structure des poèmes. Au niveau microstructural, des exemples démontrent le rôle explicatif ou réducteur d'une métaphore que joue la comparaison.

Jamais, jamais! à ceux qui s'abreuvent de pleurs

Et tètent la Douleur comme une bonne louve !

Aux maigres orphelins séchant comme des fleurs ! (Le Cygne)

Dans les deux derniers vers, le même procédé est employé : une comparaison suit un emploi métaphorique et vient l'expliciter. L'emploi du verbe «téter » est d'abord métaphorique avec un complément non animé comme «La Douleur»; le verbe est pris ensuite dans son sens premier avec le segment comparatif qui suit. D'une manière similaire, le participe présent «séchant» est métaphorique associé à «orphelins » alors qu'il retrouve son sens premier appliqué au substantif «fleurs ». Cette disposition qui force un terme à se partager entre deux acceptions, dans un même vers, l'une figurée, l'autre propre, illustre parfaitement la tension entre la vertu dénotative et imaginaire des mots. Dans le poème Le Soleil encore, deux occurrences illustrent cette tension :

Il fait s'évaporer les soucis vers le ciel,

\footnotetext{
19 C. Détrie, Du Sens dans le processus métaphorique, Paris, Champion, 2001.

20 Voir M. Murat, Poétique de l'analogie, Paris, Corti,
} 
Et remplit les cerveaux et les ruches de miel.

C'est lui qui rajeunit les porteurs de béquilles

Et les rend gais et doux comme des jeunes filles,

Et commande aux moissons de croître et de mûrir

Dans le cœur immortel qui toujours veut fleurir !

Le mot «miel » est employé à la fois avec son sens dénoté et dans un sens métaphorique, que l'on peut interpréter comme véhiculant toutes ses connotations connues et positives. La conjonction de coordination qui réunit deux substantifs sans relation sémantique évidente entre eux, recèle le potentiel métaphorique du vers. Le dernier vers oblige, quant à lui, à une lecture à rebours métaphorique du nom «moissons », qui fonctionne alors comme métaphore in absentia. Cette surprise est accentuée par l'enjambement d'un vers sur l'autre et par la reprise d'une métaphore verbale de même registre («fleurir »).

Au niveau macrostructural ensuite, la dynamique de la métamorphose du réel peut structurer tout un poème ; c'est le cas de Paysage. L'imaginaire prend le relais des notations concrètes. «L'atelier», quoique la relative implique un léger écart métonymique, est développé par les « tuyaux », les « cloches » réunis par le même sème de la verticalité, comme éléments du décor vu mais aussitôt réévalués par la métaphore appositive « ces mâts de la cité » qui occupe tout le second hémistiche, sans qu'on sache précisément s'il faut limiter l'antécédent du démonstratif anaphorique au syntagme nominal précédent. De la même façon, le syntagme pluriel «ciels » introduit à un registre pictural. La relative qui suit laisse prendre un nouvel envol à l'imaginaire déjà subrepticement annoncé par l'adjectif «grands » antéposé et évaluatif. Une analogie qu'on pourrait qualifier d'englobante fait du parcours à travers Paris une image de l'itinéraire créateur du poète. Une métaphore réduite par une comparaison encore permet d'assimiler les pavés de la ville aux mots et aux vers.

Je vais m'exercer seul à ma fantasque escrime,

Flairant dans tous les coins les hasards de la rime,

Trébuchant sur les mots comme sur les pavés

Heurtant parfois des vers depuis longtemps rêvés. (Le Soleil)

On pourrait ainsi lire la section des Tableaux parisiens comme une vaste métaphore du travail poétique qui crée à partir du monde référentiel, un monde parallèle, fictionnel, mais qui ne fait que proposer une lecture du premier. 


\section{Conclusion}

La tension entre référence et imaginaire peut se décliner en une série d'oppositions, entre le multiple et l'unicité, le particulier et l'universel, le concret et l'abstrait, l'extériorité et l'intériorité. Des instruments linguistiques font basculer des notations référentielles dans la sphère de l'imaginaire: tous ceux qui servent l'abstraction temporelle et personnelle par exemple, comme l'infinitif, le présent générique, l'impersonnel pour la catégorie verbale d'une part, les outils de la nominalisation d'autre part. Le travail de l'imaginaire est particulièrement clair lorsqu'il s'exerce sur des éléments que la langue définit comme ancrés dans une situation d'énonciation particulière, par exemple le tiroir verbal du passé simple, les déictiques comme « voici » ou encore la situation discursive de l'apostrophe. L'imagination œuvre alors pour la dilution temporelle et d'une façon générale pour l'explosion de tous les cadres qui limitent une expérience. Il ne s'agit pas cependant d'établir une rupture entre référence et imaginaire : au contraire, les ressources de la syntaxe, du lexique, de la métrique permettent de laisser apparents les points d'articulation entre l'une et l'autre. Le verbe «voir» polysémique maintient l'ambiguité permanente; le traitement du nom propre ou du nom commun qui échangent quelquefois leurs particularités ou encore l'emploi du verbe «faire » factitif ou attributif font se côtoyer en toute harmonie l'univers référentiel et l'univers imagé. La métrique et notamment les phénomènes de discordance entre mètre et syntaxe ménagent des effets de réconciliation ou accentuent les contrastes. A un niveau macrostructural enfin, le couple référence - imaginaire sert d'ossature à plusieurs pièces de la section. L'écriture poétique se propose en fait comme un acte de référenciation qui emprunte les voies détournées du fictionnel et de l'imaginaire. Elle n'est pas encore déliée du référent et à vocation autotélique comme pourra l'être l'écriture mallarméenne. 